\title{
Effect of strain rate on mechanical properties and microstructure of TRIP steel
}

\author{
Libo Pan ${ }^{1 *}$, Wanjun Zhu ${ }^{2}$, Zhaojun Deng ${ }^{2}$ and Zhijiang Zuo ${ }^{1}$ \\ ${ }^{1}$ Insititute of Intelligent Manufacturing, Jianghan University, Wuhan, China \\ ${ }^{2}$ Baosteel Central Research Institute, Wuhan, China
}

\begin{abstract}
TRIP effect is one of the important features for TRIP steel. A Nb-Mo microalloyed TRIP steel was developed by employing two different soaking time during annealing, and tensile tests at three different strain rate for specimens after annealing were performed. The microstructures and the volume fraction of retained martensite were examined and analyzed. The result indicated that TRIP steel showed significantly different behaviour at different strain rates. The ultimate tensile strength and total elongation decreased with the strain rate increasing. The dynamic strain aging effect might occur at low strain rate. With shorter soaking time during annealing, microstructure with ultra-refined grain size could be achieved. Fracture surfaces were different at different strain rate. As strain rate increases, the fraction of retained austenite to martensite transformation at failure point decreases which leads to the difference of mechanical properties.
\end{abstract}

\section{Introduction}

As a type of advanced high strength steel, TRIP steel draws lots of attention for its excellent mechanical properties combination of high tensile strength and good ductility[1-2]. It's a promising material candidate for automotive application on the purpose of light-weighting, energy-saving and safety improving. Many researchers have carried out researches on this material during recent years[3-5]. Zeng Z.L.[6] studied the mechanical properties and microstructure of a $\mathrm{Nb}$-Ti microalloyed TRIP steel, and found that the addition of trace $\mathrm{Nb}$ and $\mathrm{Ti}$ effectively refines original austenite grain size, The high density of nano-sized $(\mathrm{Nb}, \mathrm{Ti}) \mathrm{C}$ precipitates as a significant contribution on the pinning effect on dislocations improve the strength. Van H.D.[7]investigated the effects of intercritical annealing and isothermal bainite treatment parameters on macroand micro- properties of a TRIP steel and established relations between heat treatment parameters and microstructural characteristics, mechanical properties. Xu D.C. [8] revealed that the final tensile strength of TRIP steel increased and retained austenite carbon content decreased with increasing holding time. Basically one of the important features for TRIP steel is retained austenite to martensite transformation induced plasticity. In practice, the strain rate during application varies in a large range. During sheet metal forming it is ranging from $10^{-1 / \mathrm{s}}$ to10/s, and it's more than $100 / \mathrm{s}$ during car crashing. Therefore, it is necessary to investigate the relationship between the formability and the strain rate of TRIP steel, which could provide better understanding for this material and better guide for its application of a Mo-Nb microalloyed medium Mn TRIP steel.

*Corresponding author e-mail: panlb@jhun.edu.cn

\section{Material and experimental procedure}

The investigated material is a Mo-Nb microalloyed TRIP steel with medium $\mathrm{Mn}$. And it was melt by vacuum induction furnace. The chemical composition is shown in Table 1. After melting, small ingots were reheated to $1200^{\circ} \mathrm{C}$ for one hour in a protective $\mathrm{Ar}$ atmosphere and then hot-rolled to $5 \mathrm{~mm}$ in thickness, followed by oil quenching to room temperature. Small billets with the size of $75 \mathrm{~mm} \times 35 \mathrm{~mm} \times 5 \mathrm{~mm}$ were cold-rolled from $5 \mathrm{~mm}$ to about $1 \mathrm{~mm}$ by multi-passes on a small rolling mill after milling. The tensile test specimens parallel to rolling direction were milled after cold rolling. The specimens were annealed at $650^{\circ} \mathrm{C}$ for 30 minutes and 360 minutes respectively by a muffle furnace in an Ar atmosphere and then cooled in the air to room temperature. Two different soaking time 30 minutes and 360 minutes were employed to obtain different product with different average grain size.

Table 1. The main chemical composition.

\begin{tabular}{|c|c|c|c|c|c|}
\hline \multicolumn{7}{|c|}{ Chemical Composition [wt $\%]$} \\
\hline $\mathrm{C}$ & $\mathrm{Mn}$ & $\mathrm{Al}$ & $\mathrm{Nb}$ & $\mathrm{Mo}$ & $\mathrm{N}$ \\
\hline 0.167 & 6.57 & 1.1 & 0.05 & 0.22 & 0.03 \\
\hline
\end{tabular}

In this study, tensile tests are considered for the tested materials at low strain rates of $10^{-4} / \mathrm{s}, 10^{-2} / \mathrm{s}$ and $10^{-1} / \mathrm{s}$, respectively. The tensile tests at low strain rates were carried out on INSTRON $596730 \mathrm{kN}$ machine. Figure1 shows the dimension of low strain rate tensile specimen. 


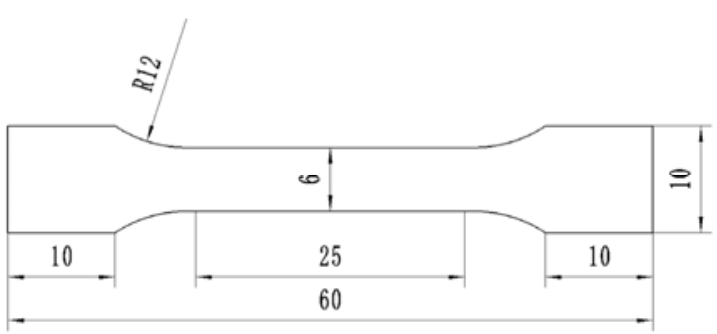

Figure 1. Dimension of tensile specimen.

The microstructure was observed by scanning electron microscope (SEM). Specimens for angle-selective backscatter (AsB) and electron backscatter diffraction (EBSD) were prepared following a standard mechanical polishing. Then samples were finished with a colloidal silica attack-polishing agent (OPS). AsB technique was utilized to obtain the detailed microstructural features, such as dislocations, twins, grain boundaries and deformation bands. The EBSD technique was employed to determine the phase fraction, crystallographic texture and interface character and substructure characteristics on a FEG Quanta 3D FEI scanning electron microscope equipped with a TSL OIM EBSD system at $30 \mathrm{kV}$ and $4 \mathrm{nA}$. Specimens for X-ray diffraction (XRD) were machined, ground and prepared metallographically in the same sequence as for electron microscopy. XRD measurements were made on a PANalytical diffractometer equipped with a $\mathrm{Cu}$ tube at a current of $30 \mathrm{~mA}$ and a voltage of $40 \mathrm{kV}$. Quantitative $\mathrm{XRD}$ analysis was performed to determine the fraction of retained austenite $V_{\gamma}$. The volume fraction of retained austenite was calculated from the integrated intensities of $(220)_{\gamma},(311)_{\gamma}$ peaks of austenite, and those of $(221)_{\alpha}$, $(200)_{\alpha}$ peaks of ferrite.

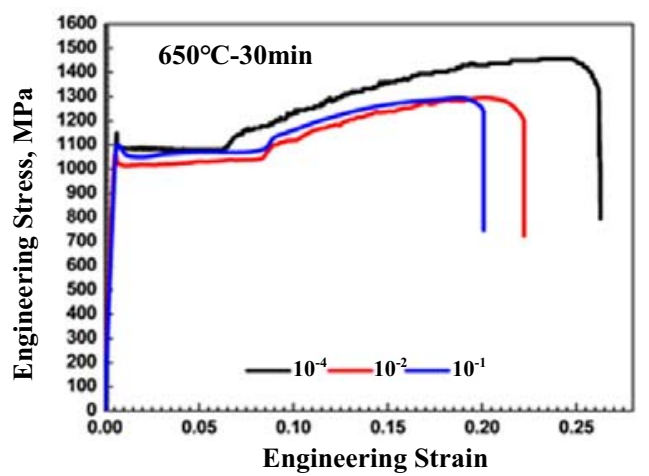

\section{Result and analysis}

\subsection{Mechanical properties}

The engineering stress-strain curves for specimens with different soaking time $30 \mathrm{~min}$ and $360 \mathrm{~min}$ during annealing are shown in Figure 2. It could be known that the strengths including yield strengths(YS) and ultimate tensile strengths(UTS) for specimens with 30min soaking time are much high than that with 360 min soaking time, while the total elongation(TE) for the former is obviously lower. For specimens with $30 \mathrm{~min}$ soaking time, YS at different strain rates ranges from $1000-1100 \mathrm{MPa}$, and UTS is in the range of $1300-1500 \mathrm{MPa}$. For specimens with $360 \mathrm{~min}$ soaking time, YS at different strain rates ranges doesn't change too much with the range from $800-830 \mathrm{MPa}$, and UTS is in the range of $1300-1500 \mathrm{MPa}$, which means soaking time during annealing has strong effect on the mechanical properties of TRIP steel. Under the condition of same soaking time, the mechanical properties also change with strain rate changing. It could be seen that the lowest YS is obtained at strain rate of $10^{-2} / \mathrm{s}$, and the highest UTS and TE could be achieved when the strain rate is $10^{-4} / \mathrm{s}$ with the strength-ductility balance(UTS $\times$ TE) up to $35 \mathrm{GPa} \%$. Generally, both UTS and TE decrease with the strain rate increasing, which indicates that for TRIP steel the strain rate apparently affects the mechanical properties, and lower strain rate is favourable to achieve higher strength-ductility balance.

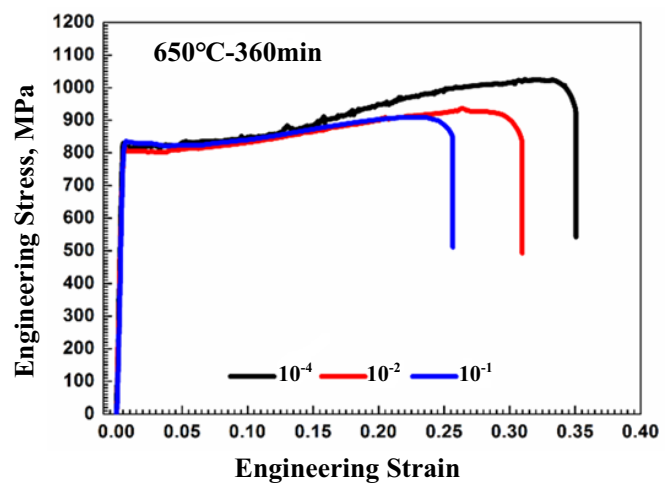

Figure 2. Engineering stress-strain curves of test specimens.

The flow behaviour for specimens with soaking time of 30minutes at different strain rates is shown in Figure 3. The work hardening rate $\mathrm{d} \sigma / \mathrm{d} \varepsilon$ and true stress-strain curves show obvious peaks near the yield points, and the $\mathrm{d} \sigma / \mathrm{d} \varepsilon$ value slightly changed as strain rate changing. As shown in Figure 2 and Figure 3, the mechanical properties of this TRIP steel are different at different strain rates.

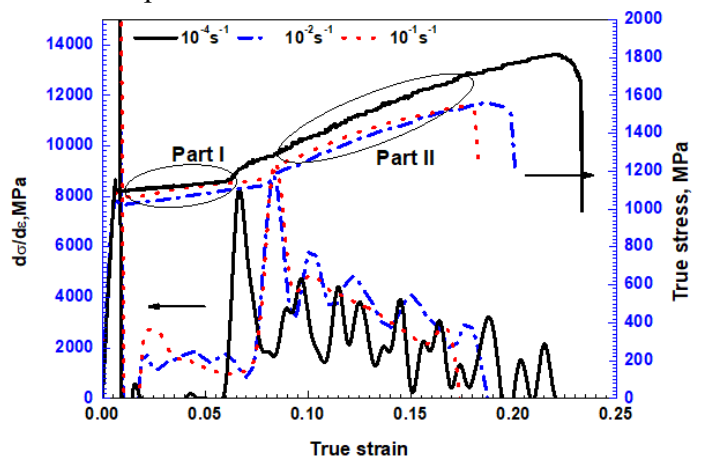

Figure 3. The $\mathrm{d} \sigma / \mathrm{d} \varepsilon$ and true stress-strain curves for specimens with 30-minute soaking time.

There is also another phenomenon occurring at lower strain rate. Figure 4 shows details of true strain curves 
with magnification in regard to part I and part II area

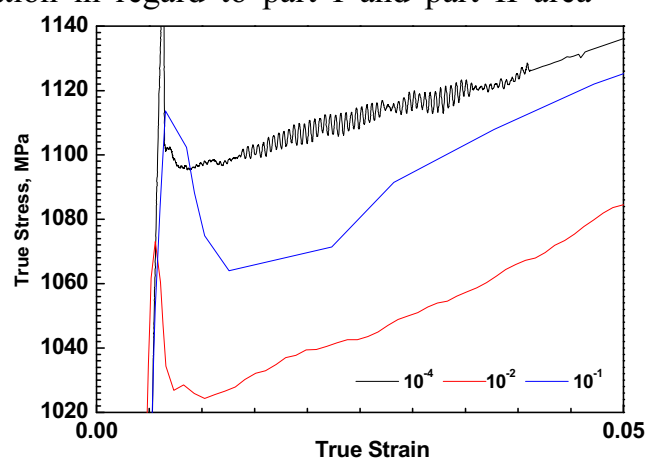

Figure 4. Partial magnification from

Apparent Serration could be observed from the curves at low strain rate of $10^{-4} / \mathrm{s}$, which is possibly due to dynamic strain aging (DSA)[9-10]. DSA is related to interactions between dislocations and solute interstitial atoms of $\mathrm{C}$ and $\mathrm{N}$ or other substitutional atoms such as $\mathrm{Cr}$ in the metal matrix. It could lead to successive locking and unlocking of dislocations and affect mechanical properties, for instance, increasing the UTS, which partially explains why the specimens at strain rate of $10^{-4} / \mathrm{s}$ exhibit much higher UTS than the others. The flow behaviour for specimens with soaking time of 360 minutes shows the same laws.

\subsection{Microstructure}
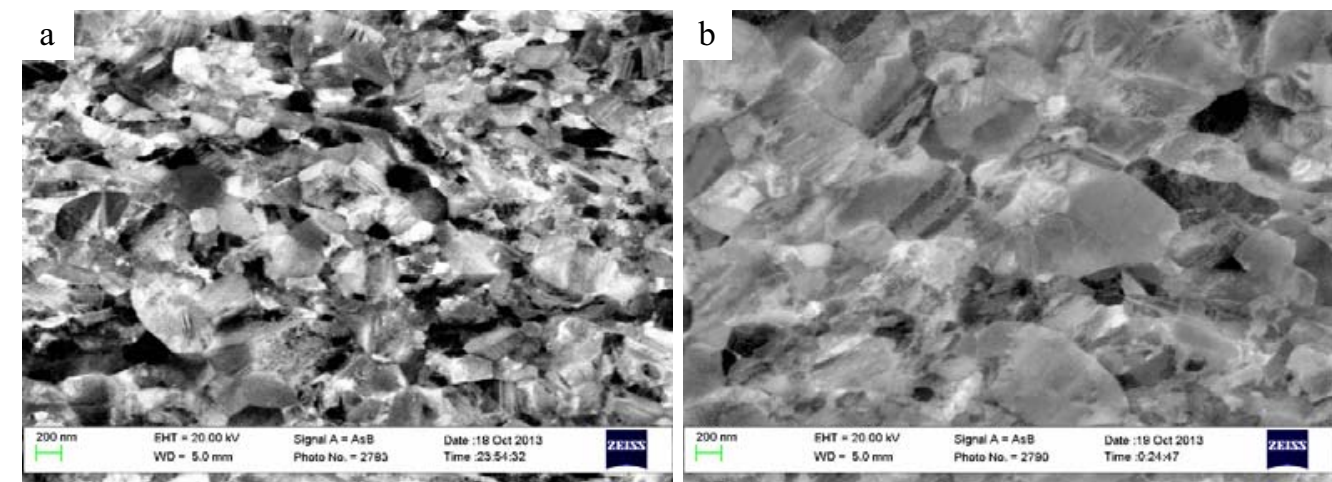

Figure 5. AsB images after annealing at $650^{\circ} \mathrm{C}$ for different soaking time: (a)30min; (b)360 min

Detailed crystallographic analysis of the ultrafine nano-sized ferrite and austenite duplex structure was performed by EBSD for the specimens annealed for 60 and $360 \mathrm{~min}$, respectively. Figure 6 shows the ferrite and austenite phase distributions. The red phase is austenite. The volume fraction of retained austenite is about $34.5 \%$ for 30-min annealed specimen and about $27.5 \%$ for

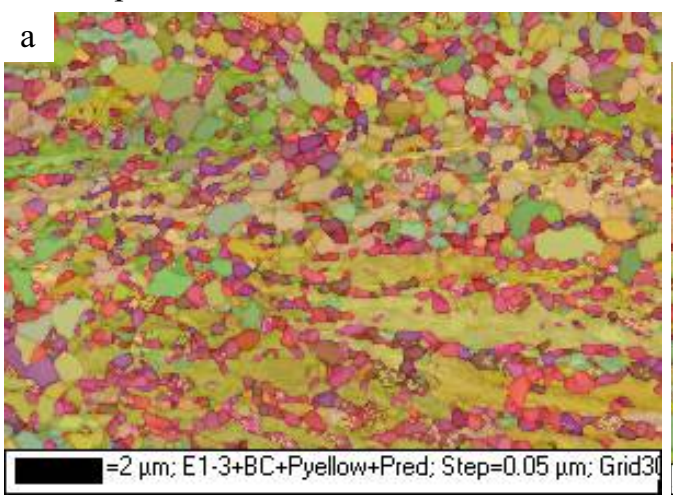

360-min annealed specimen. As demonstrated in Figure 6 (a), nucleation of $\gamma$ phase occurred preferentially at grain boundaries or grain-boundary triple points. Substantial changes in the microstructure were found after annealing for $360 \mathrm{~min}$, indicating that new $\gamma$ islands were nucleated in the ferrite grains and grew by a diffusion process leading to enrichment in $\mathrm{C}$ and $\mathrm{Mn}$ in the $\gamma$.

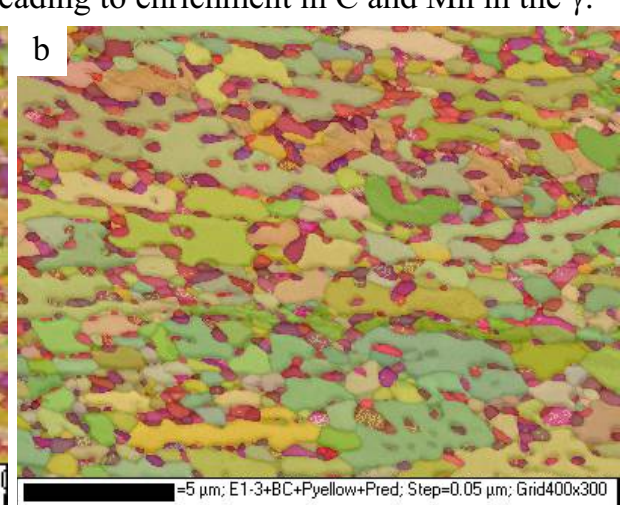

Figure 6. Phase Maps of annealed specimens, (a) 30-min, (b) 360-min.

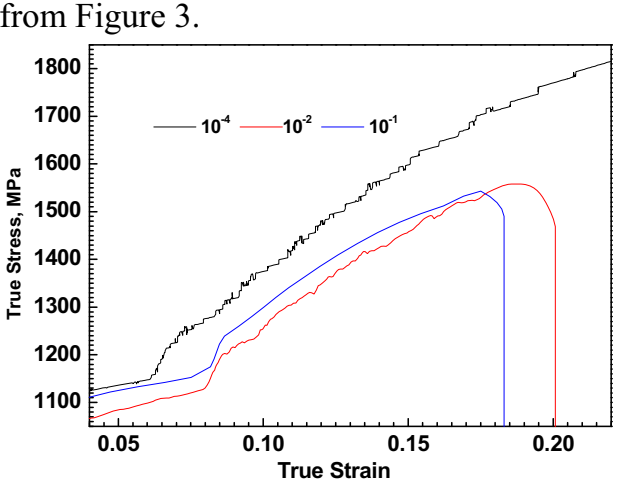

Figure 5 shows the AsB maps for samples with different soaking time. A multi-scale structure consisting of ultrafine and nano-sized equiaxed grains could be from samples with soaking time of $30 \mathrm{~min}$ ultrafine/nano duplex structure was formed during the annealing process of the cold-rolled martensite as a result competing processes of ferrite recrystallization and soaking time of 360 minutes, obvious grain growth could be observed, and the size of several coarse grains could reach up to $2-3 \mu \mathrm{m}$, which also explains why the specimens with longer soaking time got lower strengths. 


\subsection{Fracture surface observation}

Figure 7 shows the macrographs after tensile fracture for specimens with soaking time of 360 minutes at different strain rates. As shown in the figure, the laminar tearing is very obvious at low strain rate. The laminar tearing decreases as the increase of strain rates. There is no laminar tearing on the fracture surface for the samples at the strain rate of $10^{-1} / \mathrm{s}$. The macrographs of fracture surface for specimens with 30-min soaking time shows similar.
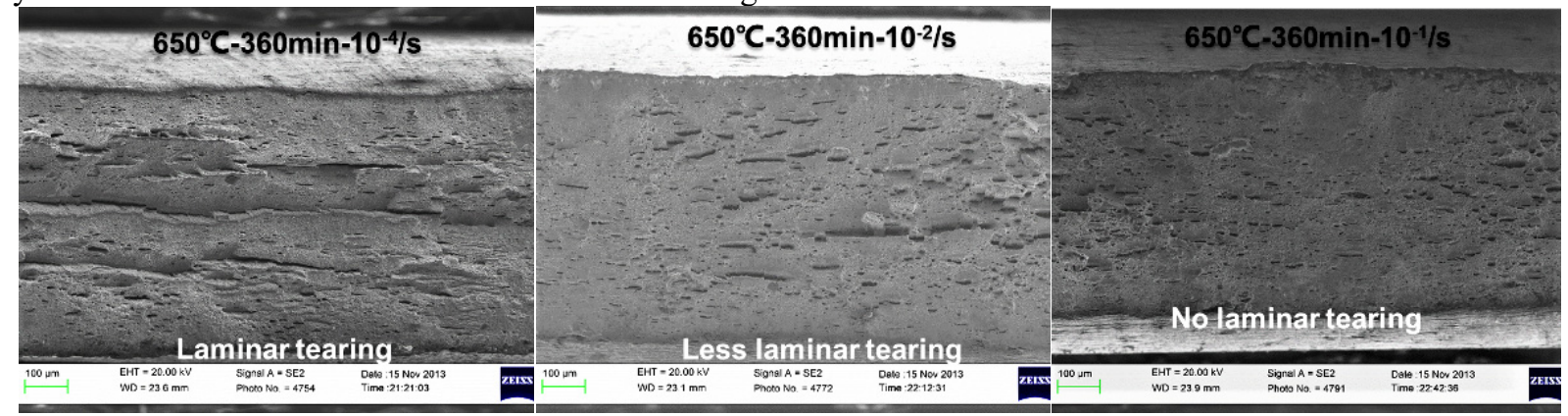

Figure 7. Macrographs of fracture surface at different strain rates

\subsection{XRD observation}

XRD tests were carried out to investigate the volume fraction of retained austenite before and after tensile deformation. As shown in Figure 8. The results for specimens with soaking time of 30 minuters at different strain rate and different strain condition were analysed. On the left side, $11 \%$ and $8.5 \%$ are specific strain and $\mathrm{F}$ means failure. The corresponding volume fraction of retained austenite $\mathrm{V}_{\gamma}$ were given on the right side. Generally the microstructure for TRIP steel consists of austenite and ferrite. Before tensile deformation, two obvious austenite peaks (220) \& (311) were observed, and the measured fraction of retained austenite is about $39.4 \%$. As TRIP effect occurred during tensile deformation, for strain rate of $10^{-4} / \mathrm{s}$, part of the retained austenite transformed to martensite at the strain point of $8.5 \%$ and the remaining $\mathrm{V}_{\gamma}$ is about $23.2 \%$. At failure point, almost all the retained austenite transformed to martensite, and no austenite peaks were observed. For strain rate of $10^{-1} / \mathrm{s}$ and $10^{-2} / \mathrm{s}$, the volume fraction of retained austenite at corresponding failure point were $19.8 \%$ and $14.3 \%$, respectively. With strain rate increasing, total elongation for specimens decreased and more austenite left without transforming to martensite, therefore, the ultimate tensile strength would be decreasing, which is in agreement with the mechanical properties result from Figure 2.The results for specimens with soaking time of 360 minuters shows similar laws.

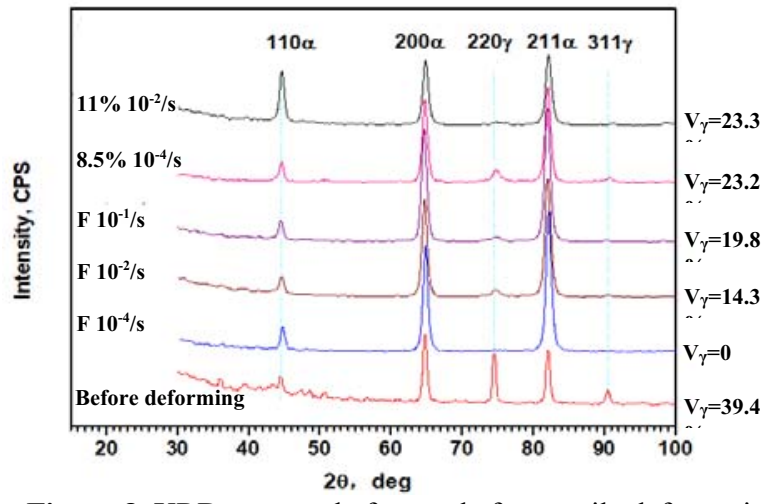

Figure 8. XRD patterns before and after tensile deformation with 30-min soaking time.

\section{Conclusion}

The studied TRIP steel annealed at $650^{\circ} \mathrm{C}$ with the soaking time of 30 minutes and 360 minutes shows a large ductility and an extremely high yield strength of more than $1 \mathrm{GPa}$ and a large strength-ductility balance (UTS $\times$ $\mathrm{TE})$ up to $35 \mathrm{GPa} \%$. The excellent strength-ductility profile was mainly attributed to grain ultra-refinement as well as the persistent strain-induced martensitic transformation during tensile deformation. Through analysis at different strain rates, the following conclusion could be drawn.

(1) The TRIP steel shows significantly different behaviour at different strain rates. The ultimate tensile strength and total elongation decrease with the strain rate increasing. The strength at the strain rate of $10^{-4} / \mathrm{s}$ is higher than the strain rate of $10^{-2} / \mathrm{s}$ and $10^{-1} / \mathrm{s}$, which is due to the dynamic strain aging (DSN). The effect of DSN on the properties at low strain rate is significant.

(2) With shorter soaking time during annealing, microstructure with ultra-refined grain size could be achieved. Fracture surfaces were different at different strain rate.

(3) As strain rate increases, the fraction of retained austenite to martensite transformation at failure point decreases which leads to the difference of mechanical properties. 


\section{Acknowledgements}

The authors would like to thank Dr. Minghui Cai from Northeastern University for indications and great support in discussing experimental procedures and understanding microstructure analysis.

\section{References}

1. Papatriantafillou I., Agoras M., Aravas N., Haidemenopoulos G. (2006) Constitutive modeling and finite element methods for TRIP steels. Comput. Methods Appl. Mech. Engrg., 195: 5094-5114

2. Ly A.L., Findley K.O. (2016) The effects of pre-straining conditions on fatigue behavior of a multiphase TRIP steel. Int. J. Fatigue, 87: 225-234.

3. Dong X.X., Shen Y.F., Xue W.Y., Jia N. (2021) Improved work hardening of a medium carbon-TRIP steel by partial decomposition of retained austenite. Mater. Sci. Eng. A, 803: 140504.

4. Kohar C.P., Cherkaoui M., Kadiri H.E., Inal K. (2016) Numerical modeling of TRIP steel in axial crashworthiness. Int. J. Plasticity, 84: 224-254.

5. Zinsaz-Borujerdi A., Zarei-Hanzaki A., Abedi H.R., Karam-Abian M., Ding H., Han D., Kheradmand N. (2018) Room temperature mechanical properties and microstructure of a low alloyed TRIP-assisted steel subjected to one-step and two-step quenching and partitioning process. Mater. Sci. Eng. A, 725: 341-349.

6. Zeng Z.L., Reddy K.M., Song S.X., Wang J.F., Wang L., Wang X.D. (2020) Microstructure and mechanical properties of $\mathrm{Nb}$ and $\mathrm{Ti}$ microalloyed lightweight $\delta$-TRIP steel. Mater. Charact., 164: 110324 .

7. Van H.D., Van C.N., Ngoc T.T., Manh T.S. (2018) Influence of heat treatment on microstructure and mechanical properties of a C-Mn-Si TRIP steel using design of experiment. Mater. Today.Proceedings, 5: 24664-24674.

8. Xu D.C., Liu Y.D., Li J., Meng Q.G., Li P. (2016) Microstructure Characterization and Mechanical Properties of TRIP aided Steel under rapid Heating for Different Holding Time. J. Iron. Steel. Res. Int., 23(2): 138-144.

9. Caillard D. (2016) Dynamic strain ageing in iron alloys: The shielding effect of carbon. Acta. Mater., 112: 273-284.

10. Nam J.H., Oh S.K., Park M.H., Lee Y.K. (2021) The mechanism of dynamic strain aging for type A serrations tensile curves of a medium-Mn steel. Acta. Mater., 206: 116613. 